\title{
Irritant contact dermatitis following extracorporeal shockwave therapy: a case report
}

\author{
Lijun Shi ${ }^{1 \#}$, Zhizhuo Li ${ }^{2 \#}$, Peixu Wang ${ }^{1}$, Meng Fan ${ }^{3}$, Wei Sun ${ }^{1,3}$ \\ ${ }^{1}$ Graduate School of Peking Union Medical College, Chin-Japan Friendship institute of Clinical Medicine, Beijing, China; ${ }^{2}$ Department of \\ Orthopedics, Peking University China-Japan Friendship School of Clinical Medicine, Beijing, China; ${ }^{3}$ Center for Osteonecrosis and Joint Preserving \\ \& Reconstruction, Department of Orthopaedic Surgery, China-Japan Friendship Hospital, Beijing, China \\ Contributions: (I) Conception and design: W Sun; (II) Administrative support: W Sun; (III) Provision of study materials or patients: L Shi, Z Li; (IV) \\ Collection and assembly of data: L Shi, Z Li; (V) Data analysis and interpretation: P Wang, M Fan; (VI) Manuscript writing: All authors; (VII) Final \\ approval of manuscript: All authors. \\ "These authors contributed equally to this work. \\ Correspondence to: Wei Sun, MD. Center for Osteonecrosis and Joint Preserving \& Reconstruction, Department of Orthopaedic Surgery, China-Japan \\ Friendship Hospital, 2 Yinghuadong Road, Chaoyang District, Beijing 100029, China. Email: cjfhsunw@163.com.
}

\begin{abstract}
Extracorporeal shockwave therapy (ESWT) has been widely used in the treatment of various musculoskeletal disorders with remarkable efficacy, with no relevant severe complications being reported. In this study, we report a rare case of acute irritant contact dermatitis following ESWT with serious skin damage, which has never been previously reported. A 42-year-old male patient with osteonecrosis of the femoral head (ONFH) was subjected to two sessions of ESWT. In the first session of low-energy ESWT $\left(0.15 \mathrm{~mJ} / \mathrm{mm}^{2}, 3 \mathrm{~Hz}\right.$, and a total of 2,000 impulses), no local or systematic adverse reactions occurred. Three months later, he was subjected to a second session of high-energy ESWT $\left(0.28 \mathrm{~mJ} / \mathrm{mm}^{2}, 4 \mathrm{~Hz}\right.$, and a total of 2,000 impulses). However, on the second day, the patient presented with itching and painful erythema and blisters on the local skin of the hip. Based on these manifestations and the results of patch testing, the patient was diagnosed with irritant contact dermatitis and treated by an oral antihistamine combined with external calamine lotions. The skin lesions began to develop exudate from erosion and scabs had gradually formed with treatment, and finally dry scabs fell off with no scar left. This is the first reported irritant contact dermatitis after ESWT. Although bone pathologies should be treated with high energy, patients should be informed of this potential rare complication.
\end{abstract}

Keywords: Contact dermatitis; extracorporeal shockwave therapy (ESWT); osteonecrosis of the femoral head $(\mathrm{ONFH})$; case report

Submitted Apr 27, 2020. Accepted for publication Nov 18, 2020.

doi: 10.21037/apm-20-1830

View this article at: http://dx.doi.org/10.21037/apm-20-1830

\section{Introduction}

Osteonecrosis of the femoral head (ONFH) is a progressive disease characterized by bone cell apoptosis and microstructural damage, which can lead to femoral head collapse and subsequent hip dysfunction (1). Currently, it has become one of the main causes of disability in the young and middle-aged. Therapy choices are mainly dependent on the staging of ONFH, and early and effective treatment before collapse is critical for delaying progression (2). Extracorporeal shockwave therapy (ESWT) uses pulsed mechanical waves which release energy when the shockwaves propagate to the interface between the bone and soft tissue. It can induce processes such as microfractures, subperiosteal hemorrhage, bone regeneration, and angiogenesis (3). Multiple studies have demonstrated the therapeutic potential of ESWT in ONFH in the early stages, the effects of which include relieving hip 
pain, shrinking the necrotic lesion, reducing the staging, and improving hip function $(4,5)$.

Besides ONFH, ESWT has also demonstrated remarkable therapeutic efficacy for many other orthopedic conditions as a non-invasive and cost-effective treatment modality for the last 20 years, leading to its current widespread application (6). However, this procedure is still prone to adverse events, and attention should be paid to the contraindications and related complications. In the orthopedic field, the most frequently reported adverse reactions are skin redness, swelling, and subcutaneous ecchymosis that can recover spontaneously (7). Osteonecrosis of the humeral head and ulnar neuropathy after ESWT have also been reported (8).

In this study, we describe a rare case of acute irritant contact dermatitis following ESWT with serious skin damage, which has not been previously reported. This rare condition might have been the result of a strong physical stimulation of ESWT which destroyed the skin barrier, alerting us to the potential danger of ESWT.

We present the following case in accordance with the CARE reporting checklist (available at http://dx.doi. org/10.21037/apm-20-1830).

\section{Case presentation}

A 42-year-old male patient was admitted to our orthopedic clinic with a 3 -week history of minor right groin pain in March 2019. He had a long history of alcoholism of more than 15 years, and was diagnosed with right $\mathrm{ONFH}$ through $\mathrm{X}$-ray, computed tomography (CT) scan, and magnetic resonance imaging (MRI) examination. Fortunately, the surface of the femoral head was still complete without collapse, and the function of the hip joint was normal on physical examination. According to the classification system of the Association Research Circulation Osseous (ARCO) (9), the femoral head was still in the early stages of "pre-collapse" and the clinical staging was ARCO IIB. He was treated with ESWT combined with oral alendronate sodium tablets (70 mg PO QW; Merck \& Co., Inc., Beijing, China), and used a crutch for partial weight bearing of the right leg.

For the ESWT procedure, the patient lay flat on the therapy couch, and the posterior skin of the hip clung to the saccule of the therapy instrument (electromagnetic, Dornier Compact DELTA II, Germany; Figure 1A). Coupling gel was then smeared uniformly at the interface between the saccule and the skin to reduce the loss of shockwave energy. The center of the saccule was focused on the right femoral head under radiographic guidance (Figure 1B). In the first session, the patient was subjected to a low-energy ESWT $\left(0.15 \mathrm{~mJ} / \mathrm{mm}^{2}, 3 \mathrm{~Hz}\right.$, and a total of 2,000 impulses) so as to avoid pain intolerance. After treatment, the groin pain was alleviated immediately, and no local or systematic adverse reactions occurred.

According to the treatment plan, the patient should have underwent a second treatment session after a week, but the patient did not follow the doctor's advice for a second treatment session because his pain was relieved. Three months later, he was subjected to a second session of high-energy ESWT $\left(0.28 \mathrm{~mJ} / \mathrm{mm}^{2}, 4 \mathrm{~Hz}\right.$, and a total of 2,000 impulses). However, on the second day, the patient presented with itchy and painful erythema of the local skin of the hip that contacted the saccule. The erythema had a clear round border, and the distribution of the skin lesions matched the shape of the saccule (Figure $2 A$ ). In addition, there were some small scattered vesicles on the edges of the swollen skin lesion. We further questioned the patient regarding his allergic history, but he indicated that he was not prone to allergy and had only come into contact with coupling gel once prior, which did not cause such skin reactions. Therefore, we inferred that this adverse reaction was not caused by the coupling gel. Further patch testing was also performed on the patient's back for 2 days, and the final reading on day 5 showed negative reactions. On the basis of his history and negative patch testing results, the patient was finally diagnosed with irritant contact dermatitis, and was treated with an oral antihistamine combined with external calamine lotions. However, the treatment effect was not obvious. Four days later, the surface of the skin lesions began to develop exudate from erosion, and scabs had gradually formed. Ten days later, the dry scabs had fallen off from the center of the skin lesions (Figure 2B).

In a recent follow-up in August 2020, the patient's condition was stable, his hip pain was relieved, and physical examination of hip joint function was normal, with no impact on his everyday activities. Imaging examinations showed that there was no progress in ONFH, the femoral head was intact and there was no collapse, and the clinical stage was still ARCO IIB stage. The skin lesions returned to normal and there was no scar residue. The patient was advised to avoid strenuous exercise.

All procedures performed in studies involving human participants were in accordance with the ethical standards of the institutional and/or national research committee(s) and with the Helsinki Declaration (as revised in 2013). Written informed consent was obtained from the patient. 

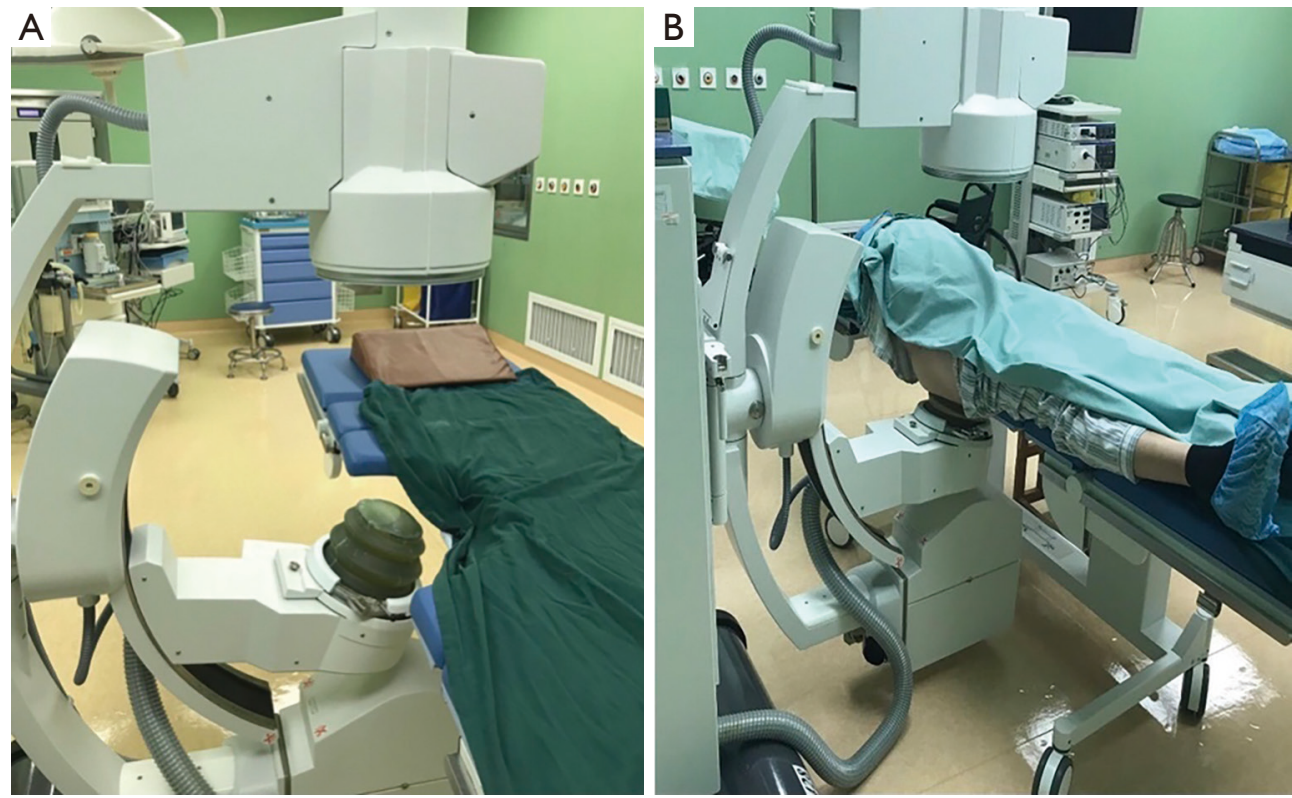

Figure 1 Device for ESWT and the operation method. (A) The ESWT instrument, which was situated in the operating room. (B) The ESWT procedure during which the posterior skin of the hip clung to the saccule of the instrument. ESWT, extracorporeal shockwave therapy.
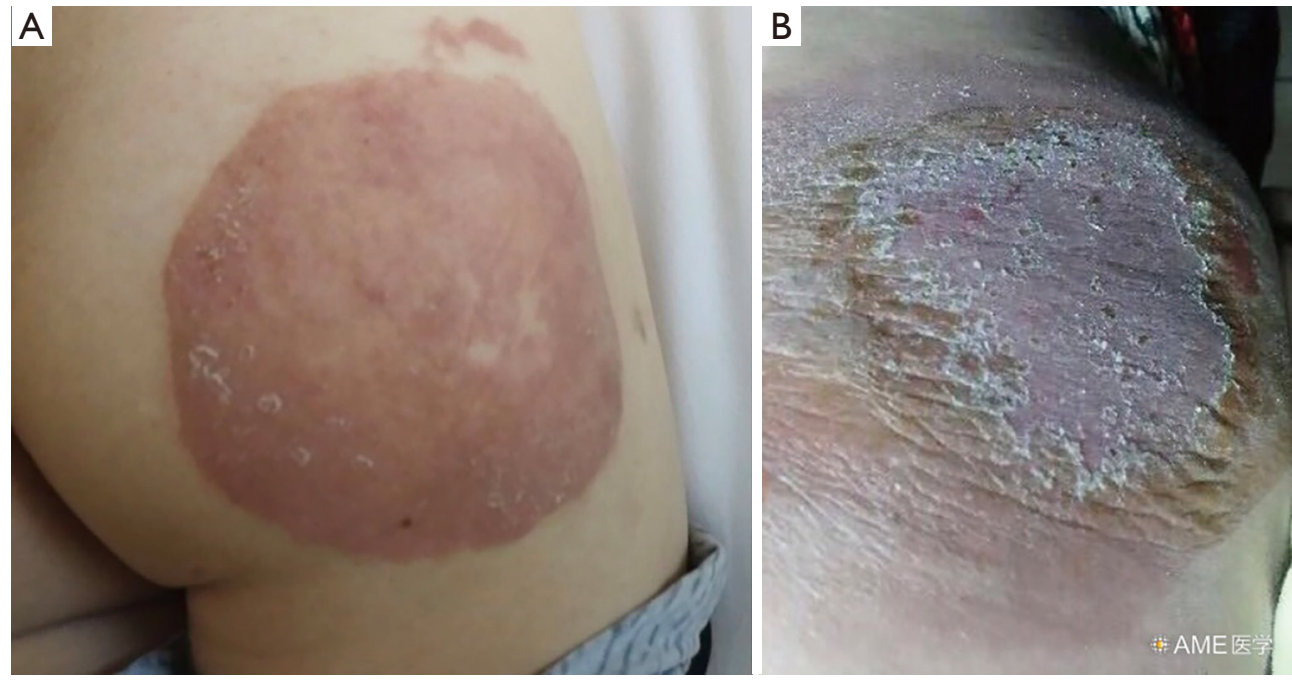

Figure 2 An unexpected skin complication after ESWT. (A) Contact dermatitis occurred on the local skin of the hip after ESWT. (B) Skin damage finally subsided after contact dermatitis. ESWT, extracorporeal shockwave therapy.

\section{Discussion}

This is the first study to report a case of irritant contact dermatitis following ESWT for the treatment of an orthopedic disorder. ESWT was first used to treat urinary calculus, although its applications have broadened in the orthopedic field in recent decades due its efficacy. As for its safety profile, it is known to be relatively safe, and the most commonly reported adverse complications are mild local skin reactions. However, other rare and serious complications have also been reported. Durst et al. (8) described a case of osteonecrosis of the humeral 
head after ESWT for the treatment of calcific tendinitis of the shoulder, which might have been due to damage of the blood supply of the humeral head. Erduran et al. (10) described a case of calcaneal stress fracture after ESWT for the treatment of plantar fasciitis. Furthermore, Lin et al. (11) described a case of calcaneal (Achilles) tendon tear after ESWT for the treatment of the chronic Achilles tendinitis. Shim et al. (12) reported another case of ulnar neuropathy after ESWT for the treatment of medial epicondylitis of the elbow. Consistent with these previous findings, this case report presents another serious complication following ESWT.

Contact dermatitis is a common dermatological disease that is usually caused by irritating chemical substances (13). However, in this current case, the patient's contact dermatitis could have been caused by physical stimulation, as the patient had previously encountered coupling gel once without any adverse reactions, and the patch testing result was negative. A rational explanation could be that the total energy of ESWT in the second session was much larger than that in the first session, which might have been beyond the tolerable range of the patient. The shockwave in this study were generated underwater through highvoltage explosion and vaporization, containing energy that is capable of causing bone microfractures (14). During propagation in the body, some of the energy is absorbed by soft tissue, which may break the skin barrier, leading to skin and mucous membrane injury. Usually, the severity of contact dermatitis is correlated with the intensity of the irritating factors. If the skin reactions are mild, they can recover without skin damage. However, the patient's case in this report was serious, and the course of skin damage was long and severe.

Potential limitations should also be considered. First, during the course of treatment, no anesthetic was used and the patient could only tolerate low energy. Hence, the patient was subjected to a low-energy ESWT in the first session. The main purpose of the first treatment session is to relieve pain, but high-energy ESWT is recommended for the treatment of ONFH. Second, the time interval between two sessions of ESWT is too long ( $>2$ weeks) for this patient. Third, we routinely used the posterior approach in the treatment procedure of ESWT for ONFH (15), which may not be the most ideal approach. The anterior approach with external rotation of the femur is recommended for the treatment of ONFH.

This case raised an important question regarding the choice of ESWT energies, as therapeutic effects are dose related. When the energy is very low, the clinical efficacy may not be satisfactory. In contrast, if the energy is too high, it may produce some undesirable complications. Considering the extensive applications of ESWT, this report is a reminder of the potential danger of contact dermatitis, and strengthens calls for more clinical trials to identify the optimal ESWT dosage for different diseases.

\section{Acknowledgments}

Funding: This study was supported by the National Natural Science Foundation of China (No. 81672236 and 82072524 , 81871830), Biomedical Translational Engineering Research Center of BUCT-CJFH (No. RZ2020-02), and the Graduate Innovation Foundation of Peking Union Medical College (No. 2019-1002-91).

\section{Footnote}

Reporting Checklist: The authors have completed the CARE reporting checklist. Available at http://dx.doi.org/10.21037/ apm-20-1830

Peer Review File: Available at http://dx.doi.org/10.21037/ apm-20-1830

Conflicts of Interest: All authors have completed the ICMJE uniform disclosure form (available at http:// dx.doi.org/10.21037/apm-20-1830) and reports grants from Beijing Natural Science Foundation, grants from National Natural Science Foundation of China, grants from Graduate Innovation Foundation of Peking Union Medical College, during the conduct of the study. The authors have no other conflicts of interest to declare.

Ethical Statement: The authors are accountable for all aspects of the work in ensuring that questions related to the accuracy or integrity of any part of the work are appropriately investigated and resolved. All procedures performed in studies involving human participants were in accordance with the ethical standards of the institutional and/or national research committee(s) and with the Helsinki Declaration (as revised in 2013). Written informed consent was obtained from the patient.

Open Access Statement: This is an Open Access article distributed in accordance with the Creative Commons Attribution-NonCommercial-NoDerivs 4.0 International 
License (CC BY-NC-ND 4.0), which permits the noncommercial replication and distribution of the article with the strict proviso that no changes or edits are made and the original work is properly cited (including links to both the formal publication through the relevant DOI and the license). See: https://creativecommons.org/licenses/by-nc-nd/4.0/.

\section{References}

1. Moya-Angeler J, Gianakos AL, Villa JC, et al. Current concepts on osteonecrosis of the femoral head. World J Orthop 2015;6:590-601.

2. Petek D, Hannouche D, Suva D. Osteonecrosis of the femoral head: pathophysiology and current concepts of treatment. EFORT Open Rev 2019;4:85-97.

3. Notarnicola A, Moretti B. The biological effects of extracorporeal shock wave therapy (eswt) on tendon tissue. Muscles Ligaments Tendons J 2012;2:33-7.

4. Algarni $\mathrm{AD}, \mathrm{Al}$ Moallem HM. Clinical and Radiological Outcomes of Extracorporeal Shock Wave Therapy in Early-Stage Femoral Head Osteonecrosis. Adv Orthop 2018;2018:7410246.

5. Xie K, Mao Y, Qu X, et al. High-energy extracorporeal shock wave therapy for nontraumatic osteonecrosis of the femoral head. J Orthop Surg Res 2018;13:25.

6. Schmitz C, Csaszar NB, Milz S, et al. Efficacy and safety of extracorporeal shock wave therapy for orthopedic conditions: a systematic review on studies listed in the PEDro database. Br Med Bull 2015;116:115-38.

7. Roerdink RL, Dietvorst M, van der Zwaard B, et al.

Cite this article as: Shi L, Li Z, Wang P, Fan M, Sun W. Irritant contact dermatitis following extracorporeal shockwave therapy: a case report. Ann Palliat Med 2021;10(6):7083-7087. doi: 10.21037/apm-20-1830
Complications of extracorporeal shockwave therapy in plantar fasciitis: Systematic review. Int J Surg 2017;46:133-45.

8. Durst HB, Blatter G, Kuster MS. Osteonecrosis of the humeral head after extracorporeal shock-wave lithotripsy. J Bone Joint Surg Br 2002;84:744-6.

9. Sugano N, Atsumi T, Ohzono K, et al. The 2001 revised criteria for diagnosis, classification, and staging of idiopathic osteonecrosis of the femoral head. J Orthop Sci 2002;7:601-5

10. Erduran M, Akseki D, Ulusal AE. A complication due to shock wave therapy resembling calcaneal stress fracture. Foot Ankle Int 2013;34:599-602.

11. Lin TC, Lin CY, Chou CL, et al. Achilles tendon tear following shock wave therapy for calcific tendinopathy of the Achilles tendon: a case report. Phys Ther Sport 2012;13:189-92.

12. Shim JS, Chung SG, Bang H, et al. Ulnar Neuropathy After Extracorporeal Shockwave Therapy: A Case Report. PM R 2015;7:667-70.

13. Novak-Bilić G, Vučić $M$, Japundžić I, et al. Irritant and allergic contact dermatitis - skin lesion characteristics. Acta Clin Croat 2018;57:713-20.

14. Romeo P, Lavanga V, Pagani D, et al. Extracorporeal shock wave therapy in musculoskeletal disorders: a review. Med Princ Pract 2014;23:7-13.

15. Gao F, Sun W, Li Z, et al. High-Energy Extracorporeal Shock Wave for Early Stage Osteonecrosis of the Femoral Head: A Single-Center Case Series. Evid Based Complement Alternat Med 2015;2015:468090. 